\title{
Population Genetics and Pharmacogenetics
}

\author{
Ken K. Ong ${ }^{a, b}$ \\ a Medical Research Council Epidemiology Unit, Cambridge, UK \\ ${ }^{b}$ Department of Paediatrics, University of Cambridge, UK
}

\begin{abstract}
A clear indication of the increasing prominence of population genetics in pediatric endocrinology is that these papers are becoming a regular feature across the other chapters of the Yearbook. For example, in other chapters this year look out for the summaries of major genetic association studies of type 1 diabetes [1-3], blood glucose levels [4,5] and bone mineral density [6], and also for an example of the emerging role of pharmacogenetics in a study of a vitamin $D$ receptor genotype [7]. In the last year, genome-wide association (GWA) studies have continued to lead on new discoveries of single nucleotide polymorphisms (SNPs) in genes or chromosomal positions (loci) that had previously not been suspected of acting in the various disease or physiological pathways. Indeed, the pace of discovery has increased with the pooling of existing data into large international consortia for meta-analyses; type 1 diabetes [1], obesity [see Willer et al. below], and blood pressure [8] are examples of such highly fruitful collaborations.

So what uses do these genetic discoveries have? There is widespread acceptance that the effect sizes of individual SNPs are much lower than were previously thought. For example, an odds ratio of $>1.3$ is now considered to be a large genetic effect, and nearly all of the 18 new SNPs for type 1 diabetes have odds ratios of $<1.2$ [1]. This means that such variants do not have, and possibly may never have, sufficient power to predict future disease risks in individuals [see Lyssenko et al. and Meigs et al. below]. New studies looking at using copy number variation or low-frequency polymorphisms may identify loci with larger effect sizes and greater ability to predict disease risks. The current discoveries have started to inform new animal models of disease, such as the Fto knockout mouse [9] [see chapter on Obesity and Weight Regulation], but it is far too early to expect any impact on drug discovery.

For the time being, recognition of new loci for common traits (e.g. age at menarche) will hopefully inform the identification of rare mutations or chromosomal microdeletions that cause severe clinical conditions (e.g. precocious puberty or hypogonadotrophic hypogonadism). In combination with international clinical collaborations, this could inform genetic counseling for affected families [see Firth et al. below]. Secondly, by Mendelian randomization principles the use of these robust variants as 'instrumental variables' to demonstrate causal effects between exposures and disease outcomes is a very powerful new epidemiological tool [see comments on Sulem et al., Perry et al., Liu et al. and Eriksson et al. below].
\end{abstract}

\section{Mechanism of the year: high throughput sequencing}

\section{Rare variants of IFIH1, a gene implicated in antiviral responses, protect against type 1 diabetes}

Nejentsev S, Walker N, Riches D, Egholm M, Todd JA

Juvenile Diabetes Research Foundation/Wellcome Trust Diabetes and Inflammation Laboratory, Cambridge Institute

for Medical Research, University of Cambridge, Cambridge, UK

Science 2009:324:387-389

Background: Genome-wide association (GWA) studies have identified genomic regions contributing to common human diseases, but they often do not identify the precise causative genes and sequence variants.

Methods: In order to identify specific causative variants for type 1 diabetes (T1D), the authors resequenced exons and splice sites of 10 candidate genes in pools of DNA from 480 patients and 480 controls and tested their association with T1D in over 30,000 participants. 
Results: Four rare variants were identified which were associated with lower T1D risk independent of each other (odds ratio $0.51-0.74 ; \mathrm{p}=1.3 \times 10^{-3}$ to $2.1 \times 10^{-16}$ ) in IFIH1 (interferon-induced with helicase $\mathrm{C}$ domain 1), a gene located in a region previously associated with T1D in GWA studies. These variants are predicted to alter the expression and structure of IFIH1.

Conclusions: These findings firmly establish the role in T1D of IFIH1 in melanoma differentiation-associated protein 5, a cytoplasmic helicase that mediates induction of interferon response to viral RNA. Furthermore, this study demonstrates that resequencing studies can pinpoint disease-causing genes in genomic regions initially identified by GWA studies.

In Yearbook 2008 we highlighted the new generation of very high-throughput DNA sequencing technologies that was used to report James Watson's DNA sequence [10]. The current paper now describes the first application of this technology in the study of complex disease. GWA studies have benefited greatly from the presence of linkage disequilibrium. This means that in many regions of our genome, numerous individual genetic variants tend to be inherited together. This means that we need to genotype 'only' a few hundred thousand variants in order to effectively 'capture' the effects of almost all the millions of common variants that exist. However the major downside is that once an association is identified, it is often not at all clear which variant or even which gene is primarily, or causally, responsible. One solution is to use large-scale gene sequencing to identify rare variants that have much larger disease associations. In contrast to the 'common' variants (with allele frequencies of $>5 \%$ ) that are typically analyzed in GWA studies, the 4 'rare' variants associated with type 1 diabetes mellitus (T1DM) in this study had allele frequencies of around $0.5-2 \%$. However, their relatively large odds ratios for association with T1DM helped to pinpoint the IFIH1 gene as responsible for the association signal on chromosome 2 q24 from the authors' previous GWA study. In essence this study now causally links IFIH1 to T1DM risk. IFIH1 is a cytoplasmic protein that recognizes RNA of picornaviruses, including enteroviruses. Enteroviral infection is more common among prediabetic and newly diagnosed T1D patients and has been suggested to trigger the appearance of autoantibodies. Upon infection, IFIH1 senses the presence of viral RNA, triggers activation of NF-кB and interferon regulatory factor pathways, and induces an antiviral interferon- $\beta$ response. Variants, which are predicted to reduce function of the IFIH1 protein, would reduce the antiviral response and decrease the risk of T1D.

\section{New paradigms: a catalog of rare events}

\section{DECIPHER: Database of Chromosomal Imbalance and Phenotype in Humans using Ensembl Resources}

Firth HV, Richards SM, Bevan AP, Clayton S, Corpas M, Rajan D, Van Vooren S, Moreau Y, Pettett RM, Carter NP Cambridge University Department of Medical Genetics, Addenbrooke's Hospital, Cambridge, UK

Am J Hum Genet 2009;84:524-533

Background: Many patients with developmental disorders have submicroscopic chromosomal deletions or duplications that, by affecting the copy number of dosage-sensitive genes or by disrupting normal gene expression, lead to disease. However, the extremely rare nature of many aberrations makes genotypephenotype correlations uncertain and therefore limits clinical interpretation. Identification of patients who share the same genomic rearrangements and similar phenotypic features enables new syndromes to be defined.

Methods: The authors have developed an interactive web-based database called DECIPHER (Database of Chromosomal Imbalance and Phenotype in Humans Using Ensembl Resources) which incorporates a suite of tools designed to aid the interpretation of submicroscopic chromosomal imbalance, inversions, and translocations.

Results: DECIPHER enhances genetic counseling by retrieving relevant information from a variety of bioinformatics resources. Known and predicted genes within a chromosomal aberration are listed in the DECIPHER patient report, and genes of recognized clinical importance are highlighted and prioritized. DECIPHER enables clinical scientists worldwide to maintain records of phenotype and chromosome 
rearrangement for their patients and, with informed consent, share this information with the wider research community through the genome browser Ensembl.

Conclusions: By sharing cases worldwide, groups of rare cases with similar phenotypes and genomic structural rearrangements can be identified. Such cooperation will facilitate the delineation of new syndromes and also further our understanding of gene function.

We often see patients with growth and developmental disorders who we think are surely 'syndromic'. However, even when our genetic analyses identify chromosomal microdeletions or other aberrations, in many cases these genomic alterations occur in chromosomal regions of unknown significance, and we are therefore no clearer to providing the family with prognostic information about their growth or future health risks. This paper reports on an international network of academic centers of clinical genetics which has developed an online tool to increase medical knowledge about microdeletions and duplications and thereby improve the medical care and genetic advice for individuals/families with these conditions (see website: https://decipher.sanger.ac.uk/). This network will also inform scientific research by providing greater certainty in the pathogenic nature of genomic rearrangements and therefore the study of genes which affect human development and health. This will be of increasing importance as high throughput sequencing techniques pick up greater numbers of potentially disruptive mutations in individual genomes.

\section{New genetic associations: a large harvest of new genes for BMI and obesity}

\section{Six new loci associated with body mass index highlight a neuronal influence on body weight regulation}

Willer CJ, Speliotes EK, Loos RJ, Li S, Lindgren CM, Heid IM, Berndt SI, Elliott AL, Jackson AU, Lamina C, Lettre G, Lim N, Lyon HN, McCarroll SA, Papadakis K, Qi L, Randall JC, Roccasecca RM, Sanna S, Scheet P, Weedon MN, Wheeler E, Zhao JH, Jacobs LC, Prokopenko I, Soranzo N, Tanaka T, Timpson NJ, Almgren P, Bennett A, Bergman RN, Bingham SA, Bonnycastle LL, Brown M, Burtt NP, Chines P, Coin L, Collins FS, Connell JM, Cooper C, Smith GD, Dennison EM, Deodhar P, Elliott P, Erdos MR, Estrada K, Evans DM, Gianniny L, Gieger C, Gillson CJ, Guiducci C, Hackett R, Hadley D, Hall AS, Havulinna AS, Hebebrand J, Hofman A, Isomaa B, Jacobs KB, Johnson T, Jousilahti P, Jovanovic Z, Khaw KT, Kraft P, Kuokkanen M, Kuusisto J, Laitinen J, Lakatta EG, Luan J, Luben RN, Mangino M, McArdle WL, Meitinger T, Mulas A, Munroe PB, Narisu N, Ness AR, Northstone K, O’Rahilly S, Purmann C, Rees MG, Ridderstrale M, Ring SM, Rivadeneira F, Ruokonen A, Sandhu MS, Saramies J, Scott LJ, Scuteri A, Silander K, Sims MA, Song K, Stephens J, Stevens S, Stringham HM, Tung YC, Valle TT, Van Duijn CM, Vimaleswaran KS, Vollenweider P, Waeber G, Wallace C, Watanabe RM, Waterworth DM, Watkins N, Witteman JC, Zeggini E, Zhai G, Zillikens MC, Altshuler D, Caulfield MJ, Chanock SJ, Farooqi IS, Ferrucci L, Guralnik JM, Hattersley AT, Hu FB, Jarvelin MR, Laakso M, Mooser V, Ong KK, Ouwehand WH, Salomaa V, Samani NJ, Spector TD, Tuomi T, Tuomilehto J, Uda M, Uitterlinden AG, Wareham NJ, Deloukas P, Frayling TM, Groop LC, Hayes RB, Hunter DJ, Mohlke KL, Peltonen L, Schlessinger D, Strachan DP, Wichmann HE, McCarthy MI, Boehnke M, Barroso I, Abecasis GR, Hirschhorn JN Genetic Investigation of Anthropometric Traits Consortium

Nat Genet 2009;41:25-34

Background: Common genetic variants at only two loci, FTO and MC4R, have been reproducibly associated with body mass index (BMI) in humans.

Methods: To identify additional reproducible loci, the authors performed a meta-analysis of 15 genomewide association studies for adult BMI $(\mathrm{n}>32,000)$. The top signals were tested for replication of association with adult BMI in 14 additional cohorts $(\mathrm{n}>59,000)$.

Results: The findings strongly confirmed FTO and MC4R and identified 6 additional loci $\left(\mathrm{p}<5 \times 10^{-8}\right)$ : TMEM18, KCTD15, GNPDA2, SH2B1, MTCH2 and NEGR1 (where a 45-kb deletion polymorphism is a candidate causal variant).

Conclusions: Several of the likely causal genes in these loci are highly expressed or are known to act in the central nervous system (CNS). These findings emphasize, as in rare monogenic forms of obesity, the likely major role of the CNS in predisposition to obesity. 


\section{Genome-wide association yields new sequence variants at seven loci that associate with measures of obesity}

Thorleifsson G, Walters GB, Gudbjartsson DF, Steinthorsdottir V, Sulem P, Helgadottir A, Styrkarsdottir U, Gretarsdottir S, Thorlacius S, Jonsdottir I, Jonsdottir T, Olafsdottir EJ, Olafsdottir GH, Jonsson T, Jonsson F, BorchJohnsen K, Hansen T, Andersen G, Jorgensen T, Lauritzen T, Aben KK, Verbeek AL, Roeleveld N, Kampman E, Yanek LR, Becker LC, Tryggvadottir L, Rafnar T, Becker DM, Gulcher J, Kiemeney LA, Pedersen O, Kong A, Thorsteinsdottir U, Stefansson K deCODE Genetics, Reykjavik, Iceland

Nat Genet 2009;41:18-24

Background: Obesity results from the interaction of genetic and environmental factors. Two common measures of obesity, weight and body mass index (BMI), are both highly heritable.

Methods: In order to identify sequence variants that influence variation in adult weight and BMI, the authors performed a genome-wide association (GWA) study with 305,846 SNPs genotyped in 25,344 Icelandic, 2,998 Dutch, 1,890 European Americans and 1,160 African American individuals. These results were combined with previously published results from the Diabetes Genetics Initiative (DGI) on 3,024 Scandinavians. 43 variants in 19 genomic regions were selected for follow-up in 5,586 Danish individuals and the results were also compared to a genome-wide study on obesity-related traits from the GIANT consortium.

Results: In total, 29 variants in 11 genomic regions reached a genome-wide statistical significance threshold of $\mathrm{p}<1.6 \times 10^{-7}$.

Conclusions: This study identified variants close to or in the FTO, MC4R, BDNF and SH2B1 genes, and also at a further 7 previously unsuspected loci, were associated with adult obesity risk.

These two papers were published back-to-back and they describe the largest GWA studies to date for adult BMI with over 25,000 individuals in the first 'genome-wide' phase of each study. Together, they identified at least 10 new loci associated with BMI. These studies confirm the principle that performing larger GWA analyses, by combining existing data from individual studies, can markedly increase the yield of true-positive signals, i.e. genetic variants that are reproducibly associated with BMI or other outcomes. By comparison, the previous GWA study of BMI based on 17,000 individuals identified only 2 loci, FTO and MC4R. Other examples of recent highly fruitful international collaborations include studies on blood pressure [8] and type 1 diabetes [1]. As more 'meta-GWA' studies appear, undoubtedly the new loci will have smaller and smaller effects. In the above papers on BMI, the effect sizes of individual variants ranged from 0.06 to 0.33 per allele, and even together they only explained a very small proportion of the variance in adult BMI. However, major benefits are the identification of new, previously unsuspected pathways to obesity, the demonstrated relevance of many of these variants also to childhood BMI and childhood obesity, and the consistent relevance of many of these BMI variants to adult type 2 diabetes risk. Most genes in these loci are expressed or are known to act in the CNS. As in rare monogenic forms of obesity, this emphasizes the major role of the CNS in predisposition to obesity. 


\section{Genome-wide association study for early-onset and morbid adult obesity identifies three new risk loci in European populations}

Meyre D, Delplanque J, Chevre JC, Lecoeur C, Lobbens S, Gallina S, Durand E, Vatin V, Degraeve F, Proenca C, Gaget S, Korner A, Kovacs P, Kiess W, Tichet J, Marre M, Hartikainen AL, Horber F, Potoczna N, Hercberg S, Levy-Marchal C, Pattou F, Heude B, Tauber M, McCarthy MI, Blakemore Al, Montpetit A, Polychronakos C, Weill J, Coin LJ, Asher J, Elliott P, Jarvelin MR, Visvikis-Siest S, Balkau B, Sladek R, Balding D, Walley A, Dina C, Froguel P CNRS 8090-Institute of Biology, Pasteur Institute, Lille, France

Nat Genet 2009;41:157-159

Background: Children with early-onset (before the age of 6 years) or adults with morbid obesity (body mass index $(\mathrm{BMI}) \geq 40$ ) may be enriched for genetic variants that influence the risk of obesity in the general population.

Methods: The authors analyzed genome-wide association (GWA) data from 1,380 Europeans with earlyonset and morbid adult obesity compared to 1,416 age-matched normal-weight controls. Thirty-eight markers showing strong association were further evaluated in 14,186 individuals of European origin. Results: In addition to FTO and MC4R, we detected a significant association of obesity with 3 new risk loci in NPC1 (endosomal/lysosomal Niemann-Pick C1 gene, $\mathrm{p}=2.9 \times 0^{-7}$ ), near MAF (encoding the transcription factor c-MAF, $\mathrm{p}=3.8 \times 10^{-13}$ ) and near PTER (phosphotriesterase-related gene, $\mathrm{p}=2.1 \times$ $\left.10^{-7}\right)$.

Conclusions: This study demonstrates the value of studying groups with early-onset, morbid adult obesity and familial recurrence to identify new susceptibility loci. The study also provides further evidence that obesity in adults and children likely share the same genetic factors.

\section{Common nonsynonymous variants in PCSK1 confer risk of obesity}

Benzinou M, Creemers JW, Choquet H, Lobbens S, Dina C, Durand E, Guerardel A, Boutin P, Jouret B, Heude B, Balkau B, Tichet J, Marre M, Potoczna N, Horber F, Le Stunff C, Czernichow S, Sandbaek A, Lauritzen T, BorchJohnsen K, Andersen G, Kiess W, Korner A, Kovacs P, Jacobson P, Carlsson LM, Walley AJ, Jorgensen T, Hansen T, Pedersen O, Meyre D, Froguel P

Genomic Medicine, Imperial College London, Hammersmith Hospital, London, UK Nat Genet 2008;40:943-945

Background: Mutations in PCSK1 cause monogenic obesity. To assess the contribution of PCSK1 to polygenic obesity risk.

Methods: The authors genotyped tag SNPs in a total of 13,659 individuals of European ancestry from 8 independent case-control or family-based cohorts.

Results: The nonsynonymous variants rs6232, encoding N221D, and rs6234-rs6235, encoding the Q665E-S690T pair, were consistently associated with obesity in adults and children $\left(\mathrm{p}=7.27 \times 10^{-8}\right.$ and $\mathrm{p}=2.31 \times 10^{-12}$, respectively). Functional analysis showed a significant impairment of the N221Dmutant PC1/3 protein catalytic activity.

Conclusions: Although additional functional analysis and replication in other cohorts will be needed, these findings firmly place PCSK1 on the short list of genes reproducibly associated with obesity.

An alternative approach to finding genes for obesity is to specifically study children with early-onset obesity or adults with morbid obesity. Such an approach had been profitable for the identification of genes for rare monogenic obesity and has now also been shown to pay off for common variants. The sample sizes required are much smaller than in studies of whole populations, however international collaborations are still needed to achieve informative numbers of severely affected individuals. However it is still unclear whether such minority groups are indeed enriched for variants that influence weight gain and susceptibility to overweight and obesity in the general population, or whether very early onset and morbid obesity are influenced by determinants that are more specific to these traits. The second study selected as a candidate the PCSK1 gene which encodes prohormone convertase $1 / 3$, the enzyme that converts prohormones into functional key hormones. Loss of function mutations of PCSK1 lead to a rare phenotype including early-onset obesity, ACTH deficiency and diabetes with an increased proinsulin/insulin ratio [11]. Frequent variants in genes that are causal for 
rare diseases have increasingly been associated with common traits (see for instance MC4R). Only 3 cases of deleterious PCSK1 mutations have yet been reported, but the findings of a robust association with common variants described here place PCSK1 on the growing short-list of genes that influence normal variations in childhood and adult obesity risk. Whether the other traits associated with the severe PCSK1 mutations will be reflected in carriers of the common variants remains to be evaluated.

\section{New paradigms: clinical phenotypes of obesity genes}

\section{An obesity-associated FTO gene variant and increased energy intake in children}

Cecil JE, Tavendale R, Watt P, Hetherington MM, Palmer CN

Bute Medical School, University of St Andrews, St Andrews, UK

N Engl J Med 2008;359:2558-2566

Background: Variation in the fat mass and obesity-associated (FTO) gene has provided the most robust associations with common obesity to date. However, the role of FTO variants in modulating specific components of energy balance is unknown.

Methods: 2,726 Scottish children, aged 4-10 years old, had weight and height measured and DNA genotyped for FTO variant rs9939609. A subsample of 97 children was examined for FTO association with adiposity, energy expenditure, and food intake.

Results: In the total group and the subsample, the A allele of rs9939609 was associated with increased weight $(\mathrm{p}=0.003$ and $\mathrm{p}=0.049$, respectively) and BMI $(\mathrm{p}=0.003$ and $\mathrm{p}=0.03)$. In the subsample, although total and resting energy expenditures were increased in children with the A allele $(\mathrm{p}=0.009$ and $\mathrm{p}=0.03$, respectively), resting energy expenditure was identical to that predicted for the age and weight of the child. Independent of body weight, the A allele was associated with increased energy intake ( $\mathrm{p}=0.006)$ but not the weight of food ingested $(\mathrm{p}=0.82)$.

Conclusions: The FTO variant does not appear to predispose to obesity by regulating lower energy expenditure. Rather it may play a role in the control of food intake and food choice, such as hyperphagia or a preference for energy-dense foods.

This is the most prominent of a number of papers over the last year describing childhood phenotypes associated with the FTO obesity susceptibility variant. On a general point, it demonstrates that while tens of thousands of individuals were required to initially identify the FTO locus [12], subsequent highly informative physiological studies of the same variants are possible in far fewer numbers if you use precise and robust methods. This study used indirect calorimetry to assess resting metabolic rate, doubly labeled water to assess total energy expenditure, and standardized test meals performed in triplicate to assess eating behavior. The findings, plus results from 2 other childhood studies, which showed associations with reduced 'satiety responsiveness' [13] and increased total energy and fat intakes [14], consistently suggest that the FTO variant influences obesity risk by central regulation of appetite. However there is a major disparity between these human associations and mouse gene knockout studies. The latter suggest that FTO leads to obesity by reducing energy expenditure and downregulation of sympathetic activation rather than increasing appetite [9]. One problem may be that the human studies have so far all been cross-sectional and it remains difficult to truly determine the causal directions between these behaviors and BMI. In addition to robust measurements, there are no shortcuts to applying robust prospective study designs to understand the genetic etiology of obesity. 


\section{Genetic determinants of height growth assessed longitudinally from infancy to adulthood in the northern Finland birth cohort 1966}

Sovio U, Bennett AJ, Millwood IY, Molitor J, O’Reilly PF, Timpson NJ, Kaakinen M, Laitinen J, Haukka J, Pillas D, Tzoulaki I, Molitor J, Hoggart C, Coin LJ, Whittaker J, Pouta A, Hartikainen AL, Freimer NB, Widen E, Peltonen L, Elliott P, McCarthy MI, Jarvelin MR

Department of Epidemiology and Public Health, Imperial College London, London, UK PLoS Genet 2009;5:e1000409

Background: Recent genome-wide association (GWA) studies have identified several common variants associated with adult height. However, it is unknown how these variants influence childhood growth. Methods: The study comprised 3,538 singletons with genotype data and frequent height measurements between 0 and 20 years (average 20 measurements/person). The authors calculated peak height velocity in infancy (PHV1) and in puberty (PHV2) and timing of pubertal height growth spurt from parametric growth curves fitted to longitudinal height growth data.

Results: 26 of the 48 known height genetic variants tested were associated with adult height $(\mathrm{p}<0.05$, adjusted for sex and principal components), reflecting the limited power to detect these associations in this dataset. 7 variants (in/near HHIP, DLEU7, UQCC, SF3B4/SV2A, LCORL, and HIST1H1D) were associated with PHV1. 5 variants (in/near SOCS2, SF3B4/SV2A, C17orf67, CABLES1, and DOT1L) were associated with PHV2. There was statistical evidence for age-dependent effects for the variants in SOCS2 $(\mathrm{p}=0.003)$ and in HHIP $(\mathrm{p}=0.045)$. None of the variants was associated with timing of the pubertal height growth spurt after correction for multiple testing.

Conclusions: This is the first genetic association analysis on longitudinal height growth in a prospective cohort from birth to adulthood. The findings support the rationale for future research on the genetic regulation of human height during different periods of growth.

This study demonstrates the very interesting potential for pediatric endocrinologists to dissect out the specific relationships of genetic variants that have recently been identified mainly for adult outcomes. Adult height is of course the sum of length at birth plus statural growth during childhood. From our understanding of the different stages of growth and modeling of these specific components [15], it should come as little surprise that genetic variants for adult height may be partitioned into subgroups that regulate specific stages. Nearly half of the genetic variants associated with adult height in this sample had a detectable effect on PHV in infancy or puberty. While they found statistical evidence for age-specific effects of two variants, the authors acknowledge that their study size lacked the power to fully explore the potential effects of many of the other variants. However, this study demonstrates the concept, and indicates that further large genetic association studies plus future characterization of the actions of these genes will undoubtedly reveal significant insights into the mechanisms that regulate the specific phases of growth and its transitions. 


\section{Genome-wide association study identifies sequence variants on 6 q21 associated with age at menarche}

Sulem P, Gudbjartsson DF, Rafnar T, Holm H, Olafsdottir EJ, Olafsdottir GH, Jonsson T, Alexandersen P, Feenstra B, Boyd HA, Aben KK, Verbeek AL, Roeleveld N, Jonasdottir A, Styrkarsdottir U, Steinthorsdottir V, Karason A, Stacey SN, Gudmundsson J, Jakobsdottir M, Thorleifsson G, Hardarson G, Gulcher J, Kong A, Kiemeney LA, Melbye M, Christiansen C, Tryggvadottir L, Thorsteinsdottir U, Stefansson K deCODE Genetics, Reykjavik, Iceland Nat Genet 2009; Epub ahead of print

Background: Earlier age at menarche (AAM) correlates with shorter adult height and higher childhood body fat. Despite a large genetic component, no common genetic variant that associates with AAM has been reported.

Methods: The authors performed a genome-wide association study of AAM in 15,297 Icelandic women. Findings were replicated in further study populations from Iceland, Denmark and the Netherlands $(\mathrm{n}=$ 10,040).

Results: Combined analyses of all study populations showed an association between $\mathrm{rs} 314280[\mathrm{~T}]$ on $6 \mathrm{q} 21$, near the LIN28B gene, and AAM (effect $=1.2$ months later per allele; $\mathrm{p}=1.8 \times 10^{-14}$ ). A second SNP within the same linkage disequilibrium (LD) block, rs314277, splits rs314280[T] into two haplotypes with different effects ( 0.9 and 1.9 months per allele). These variants have been associated with greater adult height. Other variants, previously associated with height, did not associate with AAM. Of 11 variants recently associated with higher body mass index (BMI), 5 were associated with earlier AAM.

Conclusions: Sequence variants at $6 \mathrm{q} 21$ were associated with AAM in Iceland and replicated consistently in populations from Denmark and the Netherlands. In addition, many of the SNPs that have recently been associated with increased adult BMI also associate with earlier AAM.

\section{Meta-analysis of genome-wide association data identifies two loci influencing age at menarche}

Perry JR, Stolk L, Franceschini N, Lunetta KL, Zhai G, McArdle PF, Smith AV, Aspelund T, Bandinelli S, Boerwinkle E, Cherkas L, Eiriksdottir G, Estrada K, Ferrucci L, Folsom AR, Garcia M, Gudnason V, Hofman A, Karasik D, Kiel DP, Launer LJ, van Meurs J, Nalls MA, Rivadeneira F, Shuldiner AR, Singleton A, Soranzo N, Tanaka T, Visser JA, Weedon MN, Wilson SG, Zhuang V, Streeten EA, Harris TB, Murray A, Spector TD, Demerath EW, Uitterlinden AG,

Murabito JM

Institute of Biomedical and Clinical Science, Peninsula Medical School, Plymouth, UK

Nat Genet 2009; Epub ahead of print

Background: Twin and family studies suggest a significant genetic component to menarcheal age, with at least $50 \%$ heritability.

Methods: The authors conducted a meta-analysis of genome-wide association data to detect genes influencing age at menarche in 17,510 women.

Results: The strongest signal was at $9 \mathrm{q} 31.2\left(\mathrm{p}=1.7 \times 10^{-9}\right)$, where the nearest genes include TMEM38B, FKTN, FSD1L, TAL2 and ZNF462. The next best signal was near the LIN28B gene ( $r$ 7759938; $\mathrm{p}=$ $\left.7.0 \times 10^{-9}\right)$, which also influences adult height.

Conclusions: This study provides the first evidence for common genetic variants influencing the timing of female sexual maturation.

These studies were part of a handful of GWA reports published concordantly, which describe the first common polymorphisms associated with age at menarche and also their influence on the timing of the onset of puberty and pubertal growth in both boys and girls [16, 17]. Previously, linkage and candidate gene studies had not confirmed any specific loci that were reproducibly associated with normal variation in the timing of puberty [18], and therefore these studies point to new mechanisms that regulate the timing of puberty (see chapter on Reproductive Endocrinology). In addition, the two studies described above had sufficiently large GWA datasets to explore in silico the potential effects of other variants associated with BMI and height. These results show that there are causal 
inter-relationships between the timing of puberty, BMI and adult height. For example, we had long assumed that normal variation in the timing of puberty had little effect on adult height, but very large population studies also indicate that early maturers tend to be slightly shorter [19]. The study by Sulem et al. attempted to demonstrate the direction of these relationships by adjusting the genetic associations (e.g. with adult height) for other outcomes (e.g. age at menarche), however such approaches can be biased towards the outcome which is measured with better precision. Application of Mendelian randomization principles, where genetic variants are modeled as the 'instrumental variable' that randomly changes the exposure, relies on certain assumptions [20], but provides a more robust framework to make causal inferences about the association between a risk factor and disease [21].

\title{
New genetic associations: lean body mass
}

\section{Genome-wide association and replication studies identified TRHR as an important gene for lean body mass}

\author{
Liu XG, Tan LJ, Lei SF, Liu YJ, Shen H, Wang L, Yan H, Guo YF, Xiong DH, Chen XD, Pan F, Yang TL, Zhang YP, Guo Y, \\ Tang NL, Zhu XZ, Deng HY, Levy S, Recker RR, Papasian CJ, Deng HW \\ Key Laboratory of Biomedical Information Engineering, Ministry of Education, Institute of Molecular Genetics, School \\ of Life Science and Technology, Xi'an Jiaotong University, Xi'an Shaanxi, PR China
}

Am J Hum Genet 2009;84:418-423

Background: Low lean body mass (LBM) is related to a series of health problems, such as osteoporotic fracture and sarcopenia. This paper reports a genome-wide association (GWA) study on LBM variation.

Methods: The authors performed a GWA scan using the Affymetrix 500K single-nucleotide polymorphism (SNP) array. A total of 379,319 eligible SNPs were analyzed in 1,000 unrelated US whites. Associations were replicated in 3 independent samples: (1) 1,488 unrelated US whites; (2) 2,955 Chinese unrelated subjects, and (3) 593 nuclear families comprising 1,972 US whites.

Results: Two SNPs in the thyrotropin-releasing hormone receptor (TRHR) gene were associated with LBM: $r s 16892496\left(\mathrm{p}=7.55 \times 10^{-8}\right)$ and $\mathrm{rs} 7832552\left(\mathrm{p}=7.58 \times 10^{-8}\right)$. Subjects carrying unfavorable genotypes at rs16892496 and rs7832552 had, on average, 2.70 and $2.55 \mathrm{~kg}$ lower LBM, respectively, compared to other individuals. Meta-analyses of the GWA scan and the replication studies yielded $\mathrm{p}$ values of $5.53 \times 10^{-9}$ for rs16892496 and $3.88 \times 10^{-10}$ for rs7832552. In addition, there were significant interactions between rs16892496 and polymorphisms in several other genes involved in the hypothalamic-pituitary-thyroid and the growth hormone-insulin-like growth factor-I axes.

Conclusions: These findings, together with the biological relevance of TRHR in muscle metabolism, support an important role for the TRHR gene in the regulation of LBM variation.

It is always reassuring when GWA studies identify genes that are clearly involved in known hormone pathways. It is certainly much easier to visualize the Mendelian randomization arguments linking the thyroid hormone axis to lean body mass and other outcomes. In addition, such findings may be the closest to providing new treatment strategies. These findings support a clear physiological role of the thyroid axis in promoting lean body mass. Previously, the evidence from clinical observations and trials was limited by clinical context and small numbers and the findings are inconsistent. For example, in clinical trials in hypothyroid adults, higher doses of thyroid hormone lead to greater reductions in BMI, but studies of hypothyroid patients or euthyroid volunteers showed no detectable effects of thyroxine on body composition. Furthermore, hyperthyroidism is associated with reduced muscle mass and strength. Possibly the bodybuilding fraternity could have told us these answers many years ago: 'Thyroid medication is frequently used by bodybuilders who are getting ready for a contest', and 'T4 has found a bit of a niche for those using GH and dieting' (author's undisclosed online sources). Higher doses of thyroid hormone have also been associated with improvements in ankle reflex time and working memory; Mendelian randomization studies could substantiate those findings and also suggest other effects of thyroid hormone. Finally, the growth hormone (GH) gene was 
the first to be discovered to have a thyroid response element, and the IGF1 receptor and GHRH genes were also linked to lean body mass in previous studies. The current paper indicates a thyroid-GH coregulation of lean body mass.

\section{New genetic associations: serum estrogen levels}

\section{Genetic variations in sex steroid-related genes as predictors of serum estrogen levels in men}

Eriksson AL, Lorentzon M, Vandenput L, Labrie F, Lindersson M, Syvanen AC, Orwoll ES, Cummings SR, Zmuda JM, Ljunggren O, Karlsson MK, Mellstrom D, Ohlsson C

Center for Bone Research, Sahlgrenska Academy, Department of Internal Medicine, Gothenburg University,

Gothenburg, Sweden

J Clin Endocrinol Metab 2009;94:1033-1041

Background: The risk of many conditions, including prostate cancer, breast cancer, and osteoporosis, is associated with serum levels of sex steroids. The aim of the study was to identify genetic variations in sex steroid-related genes that are associated with serum levels of estradiol $\left(\mathrm{E}_{2}\right)$, estrone $\left(\mathrm{E}_{1}\right)$ and/or testosterone in men.

Methods: The authors genotyped 604 single nucleotide polymorphisms (SNPs) in 50 sex steroid-related candidate genes in 1,041 men (age $18.9 \pm 0.6$ years) in the Gothenburg Osteoporosis and Obesity Determinants (GOOD) study. Significant associations were replicated in the Osteoporotic Fractures in Men (MrOS) Sweden study ( $\mathrm{n}=2,568$; age $75.5 \pm 3.2$ years) and in the MrOS US study $(\mathrm{n}=1,922$ men; age $73.5 \pm 5.8$ years). Serum $\mathrm{E}_{2}, \mathrm{E}_{1}$ and testosterone levels were analyzed using gas chromatography/ mass spectrometry.

Results: In the initial phase, one SNP rs2470152 in intron 1 of the CYP19 (aromatase) gene was the most strongly associated with serum $\mathrm{E}_{2}$ levels $\left(\mathrm{p}=2 \times 10^{-6}\right)$. This association was confirmed both in the MrOS Sweden study $\left(\mathrm{p}=9 \times 10^{-7}\right)$ and in the MrOS US study $\left(\mathrm{p}=1 \times 10^{-4}\right)$. In combined analyses of all populations $(\mathrm{n}=5,531)$, rs2470152 was clearly associated with both $\mathrm{E}_{2}\left(\mathrm{p}=2 \times 10^{-14}\right)$ and $\mathrm{E}_{1}(\mathrm{p}=$ $\left.8 \times 10^{-19}\right)$ levels. In addition, this polymorphism was modestly associated with lumbar spine BMD $(\mathrm{p}<$ $0.01)$ and prevalent self-reported fractures $(\mathrm{p}<0.05)$.

Conclusions: rs2470152 of the CYP19 gene, which encodes the aromatase enzyme responsible for the final step of the biosynthesis of $E_{2}$ and $E_{1}$, is clearly associated with serum $E_{2}$ and $E_{1}$ levels in men.

This paper reports a thorough candidate gene study to identify variants associated with serum estrogen and testosterone levels. It illustrates that in addition to the large GWA approach, smaller more focused and hypothesis-driven genetic studies can also be invaluable, particularly if the relatively smaller numbers allow investment in more precise phenotypic measurements, in this case more accurate hormone assays. It also illustrates the concept that identification of robust genetic markers of metabolic variables, or other specific traits, can then be used to infer causality between those variables and other clinically important outcomes. In this case, rs 2470152 was associated with lumbar spine BMD, but not femoral neck BMD, which strongly supports the notion that the lumbar spine is a more estrogen-responsive bone site than the femoral neck. The compelling evidence that $r s 2470152$ is a marker of serum $E_{2}$ and $E_{1}$ levels in men allows its use in future Mendelian randomization studies to evaluate the causal effects of normal variations in estrogen levels on various important phenotypic traits and disease risks in men. 


\title{
Interaction effect of genetic polymorphisms in glucokinase (GCK) and glucokinase regulatory protein (GCKR) on metabolic traits in healthy Chinese adults and adolescents
}

\author{
Tam CH, Ma RC, So WY, Wang Y, Lam VK, Germer S, Martin M, Chan JC, Ng MC \\ Department of Medicine and Therapeutics, Chinese University of Hong Kong, Prince of Wales Hospital, Shatin, Hong \\ Kong, SAR, China
}

Diabetes 2009;58:765-769

Background: Glucokinase regulatory protein (GCKR) is a rate-limiting factor of glucokinase (GCK), which functions as a key glycolytic enzyme for maintaining glucose homeostasis. Recent studies in European populations have reported associations between a common intronic polymorphism (rs780094) at GCKR with higher levels of triglyceride but lower fasting plasma glucose (FPG) levels and type 2 diabetes risk.

Methods: In order to examine their associations with metabolic traits in a healthy Chinese population, the authors genotyped two single nucleotide polymorphisms (SNPs), rs780094 at GCKR and rs1799884 at GCK, in 600 adults and 986 adolescents. Associations were adjusted for age, sex, and/or BMI. Interaction between these two SNPs was also tested, and a meta-analysis among European and Asian populations was performed.

Results: The T-allele of GCKR rs780094 was associated with increased triglycerides $\left(\mathrm{p}=5.4 \times 10^{-7}\right)$, while the A-allele of GCK rs1799884 was associated with higher FPG $\left(\mathrm{p}=3.1 \times 10^{-7}\right)$. A novel interaction effect between the two SNPs on FPG was also observed $(\mathrm{p}=0.0025)$. Meta-analyses strongly supported the additive effects of GCKR rs780094 and GCK rs1799884 on FPG and triglycerides, respectively.

Conclusions: In support of the intimate relationship between glucose and lipid metabolisms, GCKR and GCK genetic polymorphisms interact to increase FPG in healthy adults and adolescents. These risk alleles may contribute to increased diabetes risk in subjects who harbor other genetic or environmental/ lifestyle risk factors.

So far, the main findings of GWA studies have come from individual analyses of each genetic variant considered on its own. Subsequent analyses, for example using risk-allele or risk-genotype scores [22], have shown that these individual genetic effects do indeed appear to be simply additive. The opposite scenario, that some genes can modify the effects of other genes seems more plausible from a systems biology perspective. However, the tests for gene-gene modification (which are detected by statistical interaction or epistasis) are relatively underpowered, and in general require samples sizes around four times larger than those needed for simple associations. The results of this study only add to that complexity. The rs780094 variant in GCKR is primarily associated with higher triglyceride levels, but contrary to initial expectation, is also associated with lower glucose and insulin levels [23]. In this Chinese population, GCKR rs780094 showed no direct association with FPG levels, but modified the effect of another well-established variant GCK rs1799884 on FPG. However, no such interaction was seen on triglyceride levels. The meta-analysis found no other reports of any interactions between these two variants in Europeans, possibly due to lower allele frequencies and reduced power. This paper suggests that gene-gene interactions may be specific to each trait and also to each population, which makes their detection and validation even more challenging. 


\section{Clinical risk factors, DNA variants, and the development of type 2 diabetes}

Lyssenko V, Jonsson A, Almgren P, Pulizzi N, Isomaa B, Tuomi T, Berglund G, Altshuler D, Nilsson P, Groop L Department of Clinical Sciences, Lund University, Malmö, Sweden

N Engl J Med 2008;359:2220-2232

Background: Type 2 diabetes mellitus (T2DM) results from an interaction between environmental and genetic factors. The combination of clinical and genetic factors could predict progression to diabetes. Methods: The authors genotyped 16 single-nucleotide polymorphisms (SNPs) and examined clinical factors in two prospective cohorts of 16,061 Swedish and 2,770 Finnish subjects. T2DM developed in $2,201(11.7 \%)$ during a median follow-up of 23.5 years.

Results: Strong predictors of diabetes were: family history of the disease, increased BMI, elevated liverenzyme levels, baseline smoking status, and reduced measures of insulin secretion and action. Variants in 11 genes (TCF7L2, PPARG, FTO, KCNJ11, NOTCH2, WFS1, CDKAL1, IGF2BP2, SLC30A8, JAZF1, and HHEX) were associated with the risk of T2DM independent of the clinical risk factors. The addition of genotype information to clinical factors slightly improved the prediction of future diabetes (area under the receiver-operating-characteristic curve increased from 0.74 to $0.75 ; \mathrm{p}=1.0 \times 10^{-4}$ ).

Conclusions: Common genetic variants had a small effect on the ability to predict the future development of T2DM, compared with clinical risk factors. The value of genetic factors increased with an increasing duration of follow-up.

\section{Genotype score in addition to common risk factors for prediction of type 2 diabetes}

Meigs JB, Shrader P, Sullivan LM, McAteer JB, Fox CS, Dupuis J, Manning AK, Florez JC, Wilson PW, D'Agostino RB, Sr., Cupples LA

General Medicine Division, Department of Medicine, Massachusetts General Hospital, Boston, Mass., USA N Engl J Med 2008;359:2208-2219

Background: Multiple genetic loci have been robustly associated with the risk of type 2 diabetes mellitus (T2DM). Knowledge of these loci may allow better prediction of risk than knowledge of common phenotypic risk factors alone.

Methods: The authors genotyped single-nucleotide polymorphisms (SNPs) at 18 loci associated with diabetes in 2,377 participants of the Framingham Offspring Study. There were 255 new cases of T2DM during 28 years of follow-up. A genotype score was calculated from the number of risk alleles. Logistic regression was used to generate $\mathrm{C}$ statistics indicating how well the genotype score can discriminate the risk of T2DM when used alone and in addition to clinical risk factors.

Results: The mean $( \pm \mathrm{SD})$ genotype score was higher $17.7 \pm 2.7$ among those who developed T2DM than in the others $17.1 \pm 2.6(\mathrm{p}<0.001)$. The sex-adjusted odds ratio for diabetes was 1.12 per risk allele (95\% confidence interval 1.07-1.17). The $\mathrm{C}$ statistic was 0.534 without the genotype score and 0.581 with the score $(\mathrm{p}=0.01)$. In a model adjusted for sex and self-reported family history of diabetes, the $\mathrm{C}$ statistic was 0.595 without the genotype score and 0.615 with the score $(\mathrm{p}=0.11)$. In a model adjusted for age, sex, family history, body mass index, fasting glucose level, systolic blood pressure, high-density lipoprotein cholesterol level, and triglyceride level, the $\mathrm{C}$ statistic was 0.900 without the genotype score and 0.901 with the score $(\mathrm{p}=0.49)$. The genotype score resulted in the appropriate risk reclassification of, at most, $4 \%$ of the subjects.

Conclusions: A genotype score based on 18 risk alleles predicted new cases of diabetes but provided only a slightly better prediction of risk than knowledge of common risk factors alone.

The recent haul of highly reproducibly disease susceptibility genes or loci has raised the possibility of using DNA-based tests to help predict future disease risk in individuals from the general population. Already, consumer genomic companies, such as 23andMe, deCODE Genetics and Navigenics, are offering direct-to-consumer genome-wide scans for USD 399-2,500. Fear of genetic discrimination has lead to recent new legislation to protect individuals from use of their genetic information by insurers to deny, limit or cancel health insurance, and by employers to discriminate in the workplace. 
However, the results of these 2 studies, and also a 3rd large study from the Netherlands [24], show that currently available genetic information provides only a negligible improvement in the prediction of T2DM compared to using routinely available clinical information. In addition to T2DM, commercial DNA tests are currently also available for myocardial infarction, atrial fibrillation, glaucoma, prostate cancer and breast cancer. The strongest scientific data available are for the relatively rare breast cancer genes BRCA1 and BRCA2, which have far stronger effects on individual risks compared to common polymorphisms. However, even in that case the prediction data are based on studies only of high risk families, and the utility of positive and negative test results in the general population remains unclear. In addition to these long-term studies of disease prediction and legislation to prevent genetic discrimination, ethical issues regarding effects of genetic testing on anxiety, false assurance, and implications for other family members need to be addressed.

\title{
New concepts: overcoming your genetic predisposition
}

\section{The association of ENPP1 K121Q with diabetes incidence is abolished by lifestyle modification in the diabetes prevention program}

\author{
Moore AF, Jablonski KA, Mason CC, McAteer JB, Arakaki RF, Goldstein BJ, Kahn SE, Kitabchi AE, Hanson RL, \\ Knowler WC, Florez JC \\ Center for Human Genetic Research, Massachusetts General Hospital, Boston, Mass., USA \\ J Clin Endocrinol Metab 2009;94 449-455
}

Background: Ectoenzyme nucleotide pyrophosphatase phosphodiesterase 1 (ENPP1) inhibits insulin signaling. In a recent meta-analysis the Q allele in the K121Q (rs1044498) single nucleotide polymorphism in the ENPP1 gene was associated with increased risk of type 2 diabetes (T2DM).

Methods: The authors genotyped the ENPP1 K121Q variant in 3,548 adults at risk for T2DM who had participated in the Diabetes Prevention Program (DPP) study of lifestyle intervention or metformin vs. placebo. They explored genotype, intervention, and interactions as potential predictors of diabetes incidence.

Results: Fasting glucose and glycated hemoglobin were higher in QQ homozygotes at baseline $(\mathrm{p}<0.001$ for both). There was a significant interaction between genotype at rs1044498 and intervention under the dominant model $(\mathrm{p}=0.03)$. In analyses stratified by treatment arm, a positive association with diabetes incidence was found in Q allele carriers compared to KK homozygotes [hazard ratio (HR) 1.38; $95 \%$ confidence interval (CI) $1.08-1.76 ; \mathrm{p}=0.009]$ in the placebo arm $(\mathrm{n}=996)$. Lifestyle modification eliminated this increased risk. These findings persisted after adjustment for body mass index and race/ ethnicity. Association of ENPP1 K121Q genotype with diabetes incidence under the additive and recessive genetic models showed consistent trends (HR 1.10, 95\% CI 0.99-1.23, p = 0.08, and HR 1.16, $95 \%$ CI $0.92-1.45, \mathrm{p}=0.20$, respectively) but did not reach statistical significance.

Conclusions: ENPP1 K121Q is associated with increased diabetes incidence; the DPP lifestyle intervention eliminates this increased risk.

Firstly, this study confirms the association between the ENPP1 K121Q variant and increased risk of T2DM. However, the association was completely confined to those adults at risk for T2DM who were in the placebo arm of this 3-year diabetes prevention study. In contrast, the effect of the variant appeared to be completely abolished in those who were randomized to lifestyle intervention or metformin therapy. With the recent high-profile discoveries of robust genetic factors for many complex disease and traits it is important that we clearly communicate the public health message that genetic predisposition does not mean that disease risks are fixed and unchangeable. Similar misconceptions may arise from studies which demonstrate developmental programming by fetal or early life growth - 'determinism', either by genes or early environmental exposures, may lead to a misguided sense of fatalism. Rather, these genetic factors could potentially help to inform risk prediction [see Lyssenko et al. and Meigs et al., above] and could even help to guide the most efficient preventative strategies. For example, as this ENPP1 variant appears to confer increased risk of insulin resistance then strategies to promote insulin sensitivity, as used in this study, may be particularly effective. 


\section{Genetic compensation in a human genomic disorder}

Carelle-Calmels N, Saugier-Veber P, Girard-Lemaire F, Rudolf G, Doray B, Guerin E, Kuhn P, Arrive M, Gilch C, Schmitt E, Fehrenbach S, Schnebelen A, Frebourg T, Flori E

Department of Cytogenetics, Strasbourg University Hospital, Strasbourg, France

N Engl J Med 2009;360:1211-1216

Background: Deletions at 22q11.2 result in the DiGeorge (or velocardiofacial) syndrome. Duplications of the same $22 \mathrm{q} 11.2$ region result in a phenotype that has some features in common with DiGeorge.

Methods: Cytogenetic studies were performed for the purpose of genetic counseling in the parents of a girl with the DiGeorge syndrome, who carried a deletion at 22q11.2,

Results: The unaffected father showed an unexpected rearrangement of both $22 \mathrm{q} 11.2$ regions. He carried a $22 \mathrm{q} 11.2$ deletion on one copy of chromosome 22 and a reciprocal $22 \mathrm{q} 11.2$ duplication on the other copy of chromosome 22. Genetic compensation, which is consistent with the normal phenotype of the father, was shown through quantitative expression analyses of genes located within the DiGeorge genetic region.

Conclusions: This finding has implications for genetic counseling and represents a case of genetic compensation in a human genomic disorder.

DiGeorge syndrome is the most common microdeletion syndrome in humans, with an estimated frequency of 1 in 4,000 live births. Most cases occur sporadically, indicating that the deletion occurs recurrently in the population. More than $90 \%$ of patients with a 22 q11 deletion have the same $3-\mathrm{Mb}$ hemizygous deletion, suggesting that sequences at the breakpoints are susceptible to rearrangements. This study describes that in an unaffected father the phenotypic effects of the $22 q 11.2$ deletion were balanced by a 22q11.2 duplication on the other chromosome and resulted in normal gene expression levels in the father's lymphocytes. The authors discuss that copy number variations are highly common in the human genome and therefore compensatory copy number variations may explain why some loss-of-function mutations have low penetrance. The phenotype of a dominant loss-of-function mutation could be rescued by a reciprocal gain of a copy number variation. This finding also has major clinical relevance as this father's children would have a near $100 \%$ risk of unbalanced outcomes. The authors recommend that, for genetic counseling, genetic investigations should be performed in both parents even if they have normal phenotypes.

References

1. Barrett JC, Clayton DG, Concannon P, Akolkar B, Cooper JD, Erlich HA, et al: Genome-wide association study and meta-analysis find that over 40 loci affect risk of type 1 diabetes. Nat Genet 2009 [Epub ahead of print].

2. Smyth DJ, Plagnol V, Walker NM, Cooper JD, Downes K, Yang JH, et al: Shared and distinct genetic variants in type 1 diabetes and celiac disease. N Engl J Med 2008;359:2767-2777.

3. Cooper JD, Smyth DJ, Smiles AM, Plagnol V, Walker NM, Allen JE, et al: Meta-analysis of genome-wide association study data identifies additional type 1 diabetes risk loci. Nat Genet 2008;40:1399-1401.

4. Bouatia-Naji N, Bonnefond A, Cavalcanti-Proenca C, Sparso T, Holmkvist J, Marchand M, et al: A variant near MTNR1B is associated with increased fasting plasma glucose levels and type 2 diabetes risk. Nat Genet 2009;41:8994.

5. Prokopenko I, Langenberg C, Florez JC, Saxena R, Soranzo N, Thorleifsson G, et al: Variants in MTNR1B influence fasting glucose levels. Nat Genet 2009;41:77-81.

6. Styrkarsdottir U, Halldorsson BV, Gretarsdottir S, Gudbjartsson DF, Walters GB, Ingvarsson T, et al: New sequence variants associated with bone mineral density. Nat Genet 2009;41:15-17.

7. Jehan F, Gaucher C, Nguyen TM, Walrant-Debray O, Lahlou N, Sinding C, et al: Vitamin D receptor genotype in hypophosphatemic rickets as a predictor of growth and response to treatment. J Clin Endocrinol Metab 2008;93:46724682.

8. Newton-Cheh C, Johnson T, Gateva V, Tobin MD, Bochud M, Coin L, et al: Genome-wide association study identifies eight loci associated with blood pressure. Nat Genet 2009 [Epub ahead of print].

9. Fischer J, Koch L, Emmerling C, Vierkotten J, Peters T, Bruning JC, et al: Inactivation of the Fto gene protects from obesity. Nature 2009;458:894-898.

10. Wheeler DA, Srinivasan M, Egholm M, Shen Y, Chen L, McGuire A, et al: The complete genome of an individual by massively parallel DNA sequencing. Nature 2008;452:872-876.

11. Jackson RS, Creemers JW, Ohagi S, Raffin-Sanson ML, Sanders L, Montague CT, et al: Obesity and impaired prohormone processing associated with mutations in the human prohormone convertase 1 gene. Nat Genet 1997;16:303-306.

12. Frayling TM, Timpson NJ, Weedon MN, Zeggini E, Freathy RM, Lindgren CM, et al: A common variant in the FTO gene is associated with body mass index and predisposes to childhood and adult obesity. Science 2007;316:889-894. 
13. Wardle J, Carnell S, Haworth CM, Farooqi IS, O’Rahilly S, Plomin R: Obesity associated genetic variation in FTO is associated with diminished satiety. J Clin Endocrinol Metab 2008;93:3640-3643.

14. Timpson NJ, Emmett PM, Frayling TM, Rogers I, Hattersley AT, McCarthy MI, et al: The fat mass- and obesity-associated locus and dietary intake in children. Am J Clin Nutr 2008;88:971-978.

15. Karlberg J: On the modelling of human growth. Stat Med 1987;6:185-192.

16. Ong KK, Elks CE, Li S, Zhao JH, Luan J, Andersen LB, et al: Genetic variation in LIN28B is associated with the timing of puberty. Nat Genet 2009;41:729-733.

17. He C, Kraft P, Chen C, Buring JE, Pare G, Hankinson SE, et al: Genome-wide association studies identify loci associated with age at menarche and age at natural menopause. Nat Genet 2009 [Epub ahead of print].

18. Gajdos ZK, Butler JL, Henderson KD, He C, Supelak PJ, Egyud M, et al: Association studies of common variants in 10 hypogonadotropic hypogonadism genes with age at menarche. J Clin Endocrinol Metab 2008;93:4290-4298.

19. Onland-Moret NC, Peeters PH, van Gils CH, Clavel-Chapelon F, Key T, Tjonneland A, et al: Age at menarche in relation to adult height: the EPIC study. Am J Epidemiol 2005;162:623-632.

20. Sandhu MS, Debenham SL, Barroso I, Loos RJ: Mendelian randomisation studies of type 2 diabetes: future prospects. Diabetologia 2008;51:211-213.

21. Sheehan NA, Didelez V, Burton PR, Tobin MD: Mendelian randomisation and causal inference in observational epidemiology. PLoS Med 2008;5:e177.

22. Willer CJ, Speliotes EK, Loos RJ, Li S, Lindgren CM, Heid IM, et al: Six new loci associated with body mass index highlight a neuronal influence on body weight regulation. Nat Genet 2009;41:25-34.

23. Saxena R, Voight BF, Lyssenko V, Burtt NP, de Bakker PI, Chen H, et al: Genome-wide association analysis identifies loci for type 2 diabetes and triglyceride levels. Science 2007;316:1331-1336.

24. van Hoek M, Dehghan A, Witteman JC, van Duijn CM, Uitterlinden AG, Oostra BA, et al: Predicting type 2 diabetes based on polymorphisms from genome-wide association studies: a population-based study. Diabetes 2008;57:31223128 . 